\title{
Dexamethasone intravitreal implant for the treatment of noninfectious uveitis
}

\author{
Rebecca S Hunter \\ Ann-Marie Lobo \\ Department of Ophthalmology, \\ Massachusetts Eye and Ear Infirmary, \\ Harvard Medical School, \\ Boston, MA, USA
}

This article was published in the following Dove Press journal:

Clinical Ophthalmology

10 November 2011

Number of times this article has been viewed

\begin{abstract}
Uveitis can be a sight-threatening eye disease with significant morbidity. Corticosteroids remain the mainstay of treatment of uveitis and provide an effective treatment against ocular inflammation. However, the various modes available for corticosteroid drug delivery can carry significant ocular and systemic side effects which can limit their use in the treatment of uveitis. In an effort to avoid the damage to ocular structures that can ensue with recurrent episodes of ocular inflammation, the side effects associated with systemic steroids, and the need for repeated administration of both topical and locally injected corticosteroids, sustained-release intraocular corticosteroid implants have been developed. The dexamethasone (DEX) drug delivery system (Ozurdex ${ }^{\circledR}$; Allergan Inc, Irvine, CA), is a biodegradable intravitreal implant. This implant has been shown to be effective in the treatment of macular edema and noninfectious posterior uveitis and has been approved by the FDA for these entities. This review will highlight the current methods available for corticosteroid delivery to the eye with a particular emphasis on the DEX intravitreal implant and the evidence currently available for its use in noninfectious uveitis.
\end{abstract}

Keywords: dexamethasone implant, uveitis, macular edema, corticosteroids

\section{Introduction}

Uveitis refers to inflammation of the uveal tissues, including the iris, ciliary body, and choroid, however, other intraocular structures such as the retina and optic nerve may be involved. Uveitis is a leading cause of visual impairment and accounts for 30,000 new cases of legal blindness annually in the Western world. ${ }^{1}$ Although uveitis can affect all age populations, the mean age of patients with uveitis is 40 years old, which creates a large impact on society and healthcare.

The Standard Uveitis Nomenclature (SUN) criteria were developed to create a classification scheme that categorizes uveitis based on the primary anatomical location of inflammation. ${ }^{2}$ Under this method of classification uveitis can be divided into anterior, intermediate, and posterior. Anterior uveitis which can involve the iris, ciliary body, cornea, and sclera is the most common type of uveitis and accounts for approximately $50 \%-60 \%$ of all cases of uveitis in tertiary care centers. ${ }^{1}$ Most cases of anterior uveitis are noninfectious but the definitive etiology of anterior uveitis can only be found in $65 \%$ of patients, with the remaining cases regarded as "idiopathic". 1

Intermediate uveitis is used to denote intraocular inflammation involving the anterior vitreous and pars plana. Intermediate uveitis is the least common type of uveitis and has been found to account for 3\%-17\% of uveitis around the world. ${ }^{1}$ Given its more posterior location, patients with untreated intermediate uveitis are at increased 
risk for developing significant and sometimes permanent vision loss from cystoid macular edema. ${ }^{3}$

Posterior uveitis involves the retina and choroid but can also affect the optic nerve. It accounts for $10 \%-40 \%$ of uveitis cases, however it accounts for more visual loss than other forms of uveitis. Vision loss can be caused by cystoid macular edema, retinal detachment, subretinal fibrosis, or optic nerve damage. ${ }^{1,3} \mathrm{Up}$ to $50 \%$ of patients with posterior uveitis have an associated systemic disease. ${ }^{1}$

Standardization of the criteria for classifying uveitis by location of active inflammation has had an important impact not only on maintaining a consistent method of comparing clinical data from different research centers, but also on improving our understanding of treatment modalities and response to treatment. Numerous therapies exist for uveitis, but the most commonly utilized treatment is corticosteroids. This review will highlight the current methods available for corticosteroid delivery to the eye with a particular emphasis on the dexamethasone intravitreal implant and the evidence available for its use in noninfectious uveitis.

\section{An overview of corticosteroid therapy in uveitis}

Corticosteroids are the first-line therapy for noninfectious uveitis. The anti-inflammatory effects of corticosteroids are mediated by the cytosolic glucocorticoid receptors which increase expression of anti-inflammatory proteins. ${ }^{4}$ Glucocorticoids can also inhibit the release of proinflammatory cytokines both systemically and in the eye. There are different forms of administration of corticosteroids for the treatment of ocular inflammation: topical, local, and systemic. The use of topical corticosteroids for the treatment of anterior uveitis was first described in the $1950 \mathrm{~s}^{5}$ Since then, topical corticosteroids have become the primary form of treatment of anterior uveitis. The advantages of topical administration of steroids to the eye are numerous. Topical steroids provide direct delivery to the eye with excellent penetration into the anterior chamber. Given the relative ease of administration, topical steroids can be given at frequent intervals depending on the degree of active anterior chamber inflammation. Finally, topical steroids have minimal systemic absorption thereby reducing risk of systemic side effects. ${ }^{1,6}$ However, chronic administration of steroids, as is the case for any local steroid administration, can have significant local side effects, including cataract formation and increased intraocular pressure (IOP) with possible development of glaucoma. ${ }^{6}$ An additional limitation is that topical administration of corticosteroids does not penetrate ocular structures to adequately treat posterior forms of uveitis. Finally, it can be cumbersome for patients to administer frequent topical steroid drops especially on a long-term basis.

Local injection of corticosteroids is an important modality in the treatment of both intermediate and posterior uveitis. Corticosteroids such as methylprednisolone and triamcinolone can be administered to the eye either through a periocular sub-Tenon's injection, transeptal injection, or directly into the vitreous cavity. Direct administration of steroids to the eye has significant benefits in the delivery of medication to the posterior segment and proximity to more sensitive ocular structures such as the macula and optic nerve. Corticosteroid injections provide higher therapeutic concentrations in the posterior pole for adequate treatment of posterior segment inflammation. ${ }^{4}$ There is minimal systemic absorption following local administration of steroids, thereby avoiding the usual side effects of systemic corticosteroids. ${ }^{7}$ Local administration of corticosteroids is beneficial in patients who have unilateral ocular disease where systemic corticosteroids may not be desirable. However, the half-life of local corticosteroid administration ranges from 3-4 months and patients may require repeated injections. ${ }^{3}$ As the intraocular steroid concentration declines between injections, patients are potentially at increased risk of visual loss in between injections from recurrent inflammation. Finally, there are various risks to periocular steroid injection including inadvertent globe penetration, extraocular muscle injury, ptosis, and local skin depigmentation. ${ }^{1}$ The risks of intravitreal injection include vitreous hemorrhage, retinal detachment, and endophthalmitis. Both periocular and intravitreal corticosteroid administration also carry the common side effects seen with topical steroids: cataract formation and elevation of IOP. Both of these side effects are higher with intravitreal injections than with periocular and topical steroid administration. ${ }^{7}$ Studies have demonstrated that the risk of developing a cataract after intravitreal steroid injection is five-fold higher than the general population. ${ }^{8}$ Elevated IOP can be seen in up to $43 \%$ of patients after intravitreal steroid injections with a mean elevation of $11 \mathrm{mmHg}{ }^{8,9}$ In patients with elevated IOP, 51\% require treatment with topical IOP-lowering drops. ${ }^{8}$

Systemic administration of steroids is generally the firstline treatment for posterior noninfectious uveitis. ${ }^{3}$ Oral steroids are the most common route of systemic administration, but intravenous administration of steroids may be indicated in patients with sight-threatening inflammation. For patients with significant posterior segment inflammation, oral prednisone at $1 \mathrm{mg} / \mathrm{kg} /$ day can be initiated and then tapered as inflammation improves. Despite having significant 
anti-inflammatory effects in the eye, systemic administration of steroids, especially when used chronically, can have significant ocular and systemic side effects. Because uveitis is very often a recurrent disease, many patients may require prolonged treatment. The side effects of systemic steroids are numerous and include glucose intolerance, osteopenia, avascular necrosis, bone marrow suppression, gastric ulceration, weight gain, and psychological disturbances. ${ }^{110}$ In addition, as with all modes of steroid administration, patients on oral steroids are at increased risk of developing cataracts and elevated IOP.

\section{Intravitreal implants}

Long-term control of intraocular inflammation with minimal relapses portends a better visual prognosis for patients when compared to patients with repeated episodes of intraocular inflammation. ${ }^{10} \mathrm{~A}$ recent development in local drug delivery is surgically implantable steroid devices. These novel implants provide long-term, slow release of steroids to the eye providing sustained control of intraocular inflammation. These devices were developed based on the ganciclovir intravitreal implants that were used for the treatment of cytomegalovirus retinitis. ${ }^{11}$ Initial studies of implantable intravitreal devices used dexamethasone as the preferred steroid component, however initial animal studies demonstrated a short half-life with dexamethasone. ${ }^{12}$ Instead, fluocinolone acetonide which has $1 / 20$ the solubility of dexamethasone in aqueous and high potency, was used in a small sustained release implant. ${ }^{13}$ A fluocinolone acetonide (FA) intravitreal implant (Retisert; Bausch \& Lomb, Rochester, NY) was the first FDA-approved implantable device for the treatment of severe, noninfectious, posterior uveitis. ${ }^{11,14}$ This device is surgically placed in the vitreous cavity at the pars plana. Initial human studies revealed significant control of intraocular inflammation with the FA implant. ${ }^{15}$ Patients with noninfectious posterior uveitis treated with the implant had significantly decreased recurrences of inflammation, from a $62 \%$ recurrence rate pretreatment to $4 \%, 10 \%$, and $20 \%$ recurrence rates at 1,2 , and 3 years following implantation; recurrence rates of inflammation in eyes receiving the FA implant were significantly lower than in fellow nonimplanted eyes. ${ }^{15,16}$ The FA implant, which contains $0.59 \mathrm{mg}$ of fluocinolone acetonide, slowly releases corticosteroid over the course of 30 months. ${ }^{16}$ This sustained release provides significantly prolonged antiinflammatory effects with the rare need for additional local therapy. If inflammation recurred after the 30 month time point, a second FA implant could be placed adjacent to the original implant along the pars plana. ${ }^{17}$ This tandem placement avoids the need to remove the original implant which has an increased risk of vitreous or retinal hemorrhage, retinal traction, and possible development of retinal detachment.

\section{Complications of the fluocinolone acetonide implant}

The ocular complications of all corticosteroids (both local and systemic) are the same and have been discussed. These side effects are directly related to the strength of the steroid and its effective ocular penetration. Rates of adverse ocular side effects such as cataract formation and elevated IOP are higher with fluocinolone acetonide when compared to dexamethasone or triamcinolone acetonide. ${ }^{18,19}$ It is well established that corticosteroids induce molecular changes in the trabecular meshwork and lens that can then lead to steroidinduced ocular hypertension and cataract formation. However, currently there is no adequate measure to rank corticosteroids by rate of adverse events, including glucocorticoid activity, aqueous solubility, or pharmokinetics. ${ }^{20}$ To determine whether adverse effects may be related to partitioning of drug molecule into the trabecular meshwork and lens, a recent study by Thakur et al analyzed the lipophilicity of these corticosteroids and the relationship to ocular tissue partitioning in bovine and human tissue models. ${ }^{21}$ This study demonstrated that the lipophilicity of corticosteroids can be directly related to the degree of ocular side effects in animal models. The more lipophilic corticosteroid, fluocinolone acetonide, exhibits higher partitioning in the trabecular meshwork and lens in both bovine and human eye tissue which may provide one explanation why the incidence of side effects in prior clinical trials is highest with fluocinolone acetonide when compared to triamcinolone or dexamethasone. Although this study offered some supportive molecular evidence for the rate of ocular adverse effects seen in the various ocular corticosteroid preparations, there are certainly other characteristics such as length of implant duration and dose that must also be considered and have not been systematically studied.

Significant ocular adverse effects have been documented with the FA intravitreal implant. The FA implant has consistently shown marked induction of cataract formation; long-term clinical studies revealed that within 3 years of implantation, $100 \%$ of patients required cataract extraction. ${ }^{15}$ In addition, at 34 weeks $51.1 \%$ of eyes with the implant required more topical pressure-lowering agents than nonimplanted eyes and $78 \%$ of implanted eyes required pressure lowering drops by 3 years postimplantation. 
Approximately $40 \%$ of patients with the FA implant required glaucoma filtering surgery within 3 years following implantation due to uncontrolled IOP elevations. ${ }^{18}$ Unlike topical and systemic steroids which can be discontinued in the setting of significant ocular morbidity, the FA implant is not biodegradable and must be surgically removed if clinically indicated in the setting of significant adverse effects. In addition, unlike periocular and intravitreal steroid injections which generally have a half-life of 4 months or less, the FA implant has effects for up to 2.5-3 years. Patients who will be undergoing placement of the FA implant should be aware and understand the possible longterm implications of treatment with additional medications (anti-glaucoma medications) and surgery (both for cataract formation and glaucoma). Other less common side effects of the FA implant which were noted in clinical trials included hypotony $(6.1 \%)$, retinal detachment $(2.9 \%)$, endophthalmitis $(0.4 \%)$, and need for explantation at 2 years in $3.6 \%$ of cases for the following reasons: postoperative hypotony, uncontrollable IOP, endophthalmitis, and an unrelated case of intraocular lymphoma.

\section{Intravitreal dexamethasone drug delivery system Design and pharmacology}

Dexamethasone is a widely used corticosteroid for the treatment of systemic inflammatory conditions. Dexamethasone is five times more potent than triamcinolone acetonide, which is commonly used for periocular injections $\left(\mathrm{Kenalog}^{\circledR}\right)$ and intravitreal injections (Triesence ${ }^{\circledR}$ ). In addition, given its hydrophilicity, it allows for higher vitreous concentration than triamcinolone acetonide. The drawback of developing a topical or periocular dexamethasone formulation for ocular inflammatory disease has been the requirement of substantially higher concentrations of the drug to cross the cellular junctions at the ocular surface and through the blood-retinal barrier which can cause significant toxicity. ${ }^{19}$ Dexamethasone has been used as an intravitreal injection, but it has been shown to be significantly shorter acting in the vitreous than triamcinolone acetonide and fluocinolone acetonide, with a half-life of between 3-6 hours (Table 1)., ${ }^{1,20}$ This brief halflife precludes the use of dexamethasone injections as a longterm treatment for ocular inflammation. These shortcomings have been addressed with the development of a biodegradable dexamethasone drug delivery system $\left(\right.$ Ozurdex $^{\circledR}$, Allergan, Inc, Irvine, CA) (Figure 1).

The idea of a creating a biodegradable polymer matrix to be used as a sustained drug delivery system in humans
Table I Pharmacokinetics of intraocular steroids

\begin{tabular}{llll}
\hline Steroid & $\begin{array}{l}\text { Water } \\
\text { solubility } \\
(\mu \mathrm{g} / \mathrm{mL})\end{array}$ & $\begin{array}{l}\text { Half-life } \\
\text { (solubilized) }^{\mathrm{a}}\end{array}$ & $\begin{array}{l}\text { Relative } \\
\text { potency }\end{array}$ \\
\hline Triamcinolone acetonide & 21 & I8 days & $\mathrm{I}$ \\
Fluocinolone acetonide & 50 & Data not available & $0.4 \times$ \\
Dexamethasone & 100 & 3.5 hours & $3-5 \times$ \\
\hline
\end{tabular}

Note: aHalf-life in human vitreous.

has been attaining significant importance in biomedical research. These polymers have been used to create nanoparticles and microparticles for systemic drug delivery that can be targeted to specific human tissues such as the heart, or have novel peptide coatings which can be directed towards human malignancies such as intracranial tumors. ${ }^{22}$ Having a biodegradable implant as a platform for drug delivery is ideal, particularly for ocular tissues, given the small size of the organ and significant risk in placing and removing permanent implants as was discussed with the FA implant. The sustained-release dexamethasone drug delivery system is made of a solid biodegradable polymer composed of apolylactic acid-co-glycolic acid (PLGA) matrix. ${ }^{23}$ The PLGA polymer matrix dissolves completely in vivo into its components, lactic acid and glycolic acid. These products are in turn converted to carbon dioxide and water which can be easily eliminated by ocular tissue..$^{24,25}$ The biodegradable matrix is saturated with $0.7 \mathrm{mg}$ of dexamethasone. As the polymer dissolves, dexamethasone is slowly released into its target tissues, the vitreous and retina. The implant was not only developed to be biodegradable but was also designed to be administered as an officebased procedure. The implant can be injected through a 22-gauge injecting applicator through the pars plana similar to other intravitreal injections. This application is performed under a sterile technique via a bi-planar injection.

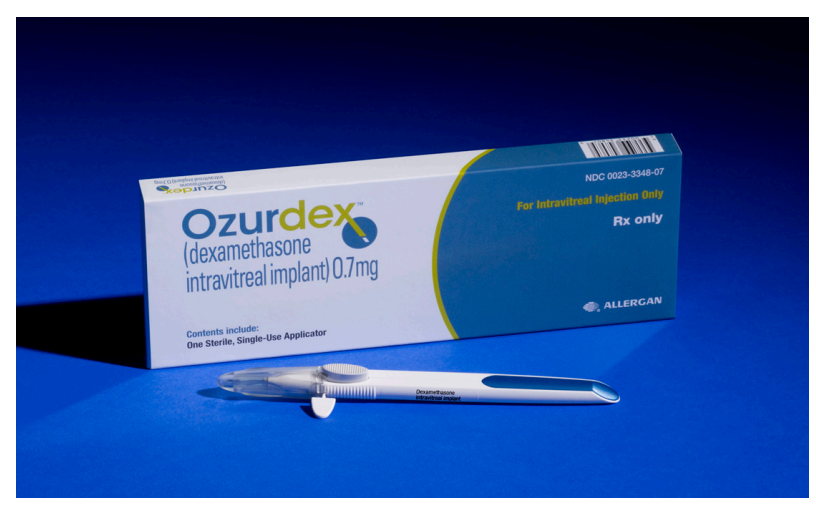

Figure I Dexamethasone intravitreal implant and injector (Ozurdex ${ }^{\circledR}$, Allergan, Inc, Irvine, CA). 


\section{Pharmacokinetics/pharmacodynamics of dexamethasone implant}

Chang-Lin et al recently determined the pharmokinetics and pharmacodynamics of the sustained-release dexamethasone drug delivery system by using mass spectrometry and expression of the dexamethasone-sensitive gene cytochrome P450 A38 (CYP3A8), which is a marker of dexamethasone biological activity in the retina. ${ }^{24}$ Thirty-four male monkeys received bilateral $0.7 \mathrm{mg}$ dexamethasone implants; three animals served as controls. Samples of blood, vitreous, and retina were retrieved up to 270 days (9 months) after initial implantation. Dexamethasone was detectable in retina and vitreous for 6 months after administration with peak concentrations during the first 2 months. The peak concentration of dexamethasone in the retina reached $1110 \pm 284 \mathrm{ng}$ and $213 \pm 49 \mathrm{ng} / \mathrm{mL}$ in the vitreous at 2 months (day 60). There was minimal systemic absorption with a peak concentration of $1.11 \mathrm{ng} / \mathrm{mL}$ in the serum at 2 months. Following the first 2 months there was a steady decline in steroid concentration from month 2 to month 3 , after which a second steady state was achieved and maintained until month 6 . The CYP3A8 expression increased more than three-fold in eyes that had received the implant compared to control eyes and this increased expression was sustained for 6 months. This study confirmed what was noted in clinical trials: the dexamethasone implant has persistent effects on target tissue up to 6 months after implantation. In addition, it was noted that the initial burst release of dexamethasone from the implant upon administration reaches an initial steady state at therapeutic concentration, which is then followed by decline to a second steady state with lower sustained release of dexamethasone. These results also confirmed the low risk of systemic absorption of medication.

\section{Clinical efficacy, safety, and tolerability of dexamethasone intravitreal implants Preclinical studies: animal models}

A non-biodegradable dexamethasone implant was first developed by Cheng et al for the treatment of experimental uveitis. ${ }^{26}$ Implantable pellets contained $5 \mathrm{mg}$ of dexamethasone and were coated in polymers to ensure sustained release of medication. These devices were implanted in rabbit eyes via a $3-\mathrm{mm}$ sclerotomy site $3 \mathrm{~mm}$ from the limbus. The device was inserted into the vitreous and sutured into place, with fellow eyes receiving implantation of polymer alone and used as controls. The rabbits were induced to have two episodes of severe panuveitis separated by 2 weeks. The implants were explanted at 99 days and found to have $30 \%$ of the drug remaining. Electroretinography (ERG) performed showed significant depression in untreated eyes compared to treated eyes. In addition, clinical findings noted significantly less inflammation in treated eyes when graded by two masked observers. The authors concluded that intravitreal implantation of a sustained-release dexamethasone device provided effective suppression of severe ocular inflammation for more than 3 months and allowed for prevention of ocular complications from recurrent inflammatory episodes in animal models. This study provided the initial support for the use of dexamethasone as a treatment for posterior uveitis.

\section{Early clinical trials: treatment of macular edema}

Initial clinical studies were performed to evaluate the safety and efficacy of the dexamethasone implant in patients with persistent macular edema. Subsequent studies would be performed to evaluate use in posterior uveitis. Kuppermann et al performed a multi-center, Phase II trial enrolling patients with persistent macular edema. ${ }^{27}$ The inclusion criteria included persistent clinically significant macular edema for 90 days or more despite laser and medical treatment. The underlying cause of macular edema could be diabetic retinopathy, retinal vein occlusion, postoperative complications such as Irvine-Gass syndrome, or uveitis. A total of 315 patients were enrolled with best-corrected visual acuity (BCVA) of 20/40 to 20/200 in the study eyes. The patients were randomized to observation, treatment with surgically implanted dexamethasone implant of two different concentrations, $0.35 \mathrm{mg}$ and $0.7 \mathrm{mg}$ in a 1:1:1 ratio. The primary outcome measure was improvement of BCVA of ten or more letters at 90 days. A significantly greater proportion of patients who received either dexamethasone implant achieved ten or more letters of visual improvement 90 days after implantation (24\% in $0.35 \mathrm{mg}$ group and $35 \%$ in the $0.7 \mathrm{mg}$ group) than control eyes (13\%). In addition, patients receiving the higher concentration implant had a greater rate of visual improvement ( $35 \%$ in $0.7 \mathrm{mg}$ implant vs $24 \%$ in $0.35 \mathrm{mg}$ implant). These results were similar in all patients despite the etiology of their macular edema. Patients were observed a total of 180 days and continued to show a statistically significant improvement in BCVA in the $0.7 \mathrm{mg}$ dexamethasone implant group compared to the control group. $11 \%$ of patients treated with the dexamethasone implant had an increase in IOP of $>10 \mathrm{mmHg}$ or higher compared to the 
control group (2\%). None of these patients required laser or surgical intervention for IOP control and were managed successfully with medication. Finally, there was no significant difference in the number of reported cataracts among the different study groups. The authors concluded that the dexamethasone implant was well tolerated and had a satisfactory safety profile.

Further subset analysis was performed examining effectiveness of treatment with the dexamethasone implant in patients with macular edema from Irvine-Gass syndrome and uveitis. ${ }^{28}$ This analysis confirmed that patients with persistent macular edema from these entities had a significant improvement in BCVA at 90 days with the dexamethasone implant. It was found that $41.7 \%$ of patients in the $0.35 \mathrm{mg}$ group and $53.8 \%$ of patients in the $0.7 \mathrm{mg}$ group had a tenletter or more improvement in BCVA (observation was $14.3 \%$ ). Furthermore, a 15-letter improvement in BCVA was achieved in $53.8 \%$ of patients receiving the $0.7 \mathrm{mg}$ dexamethasone implant. These findings were supported by a decrease in fluorescein leakage in treated patients compared to control patients. The adverse effects in this subgroup analysis of IOP increase and cataract formation were equivalent to the adverse events in the overall study.

The findings of this earlier study were confirmed with a multi-center, randomized, controlled clinical trial comparing $0.7 \mathrm{mg}$ dexamethasone implant with sham in eyes with macular edema associated with retinal vein occlusion. ${ }^{29}$ A total of 1267 patients with vision loss from macular edema secondary to a retinal vein occlusion were randomized 1:1:1 ratio to either sham procedure or treatment with dexamethasone intravitreal implant at $0.35 \mathrm{mg}$ dose and $0.7 \mathrm{mg}$ dose. The study demonstrated that the percentage of eyes achieving 15-letter improvement was significantly greater in the both dexamethasone treated groups compared to sham. The greatest response was seen at day 60 in the $0.7 \mathrm{mg}$ implant group with $29 \%$ of patients achieving 15-letter improvement. Significant adverse events experienced in the dexamethasone implant treatment groups were eye pain, ocular hypertension, and anterior chamber cellular reaction. There was no statistically significant increase in cataract formation in the treated group. $16 \%$ of implanted eyes had an increase in IOP that was greater than $15 \mathrm{mmHg}$. Most of these episodes of ocular hypertension were transient and there was no difference in IOP elevation between sham and treatment groups at 180 days. However, unlike the previous clinical trials, where no eyes required surgical or laser treatment for elevated IOP, five eyes in the dexamethasone implant groups (three in the $0.7 \mathrm{mg}$ group and two in the $0.35 \mathrm{mg}$ group) required a procedure to lower pressure. The authors cautioned that one of these eyes required the procedure for neovascular glaucoma and not for steroidinduced glaucoma. They concluded that the dexamethasone implant is a therapeutic alternative for patients with vision loss due to retinal vascular occlusion-induced macular edema. This study led to FDA approval of the dexamethasone implant for the treatment of macular edema associated with retinal vein occlusion.

\section{Dexamethasone intravitreal implant for the treatment of uveitis: clinical trials}

More recent clinical studies have focused on the use and safety of the dexamethasone implant as monotherapy for the treatment of noninfectious intermediate and posterior uveitis. ${ }^{30}$ Lowder et al evaluated the efficacy of two doses of dexamethasone (DEX) intravitreal implant in patients diagnosed with noninfectious intermediate or posterior uveitis. ${ }^{30}$ Inclusion criteria included patients with a vitreous haze score greater than +1.5 (on a scale of $0-4$ ) and a BCVA of 20/32 to 20/630. Patients were allowed to use certain topical and systemic medications during the trial if they had been stable on the medication prior to randomization. Important exclusion criteria included prior unresponsiveness to corticosteroids, glaucoma, ocular hypertension, a known history of corticosteroid-induced ocular hypertension, or uncontrolled systemic disease. Patients with any known prior use of an intravitreal device (dexamethasone or fluocinolone acetonide) were excluded. A total of 229 patients were randomized in $1: 1: 1$ ratio to receive sham procedure or treatment with dexamethasone implant (either $0.35 \mathrm{mg}$ or $0.7 \mathrm{mg}$ ). Eighty-one percent of patients in the study had intermediate uveitis. Patients were observed for 26 weeks for therapeutic effect and safety profile. The main outcome measure was the percentage of patients with vitreous haze score of 0 at 8 weeks. The proportion of patients with vitreous haze score of 0 at 8 weeks was $47 \%, 36 \%$, and $12 \%$ for the $0.7 \mathrm{mg}$ dexamethasone implant, the $0.35 \mathrm{mg}$ implant, and the sham group, respectively. The response peaked at week 8 but was maintained for up to week 26. Although not used as a main outcome measure, BCVA was significantly improved by 2-6-fold greater in the dexamethasone treated eyes than the sham group throughout the study period. The mean decrease from baseline central macular thickness on ocular coherence tomography (OCT) was also significantly greater in the dexamethasone treated groups compared to the sham group at 8 weeks. Less than $10 \%$ of eyes had an IOP of $25 \mathrm{mmHg}$ or greater. Throughout the duration of the study, 
$23 \%$ of eyes in the $0.7 \mathrm{mg}$ DEX implant group required IOPlowering medications. Most patients were either observed or treated with 1 IOP-lowering topical medication. No eyes required surgical or laser therapy for elevated IOP. Cataract formation was reported as an adverse event in $15 \%$ of eyes in the $0.7 \mathrm{mg}$ DEX implant group, $12 \%$ of eyes in the $0.35 \mathrm{mg}$ DEX implant group, and 7\% in the sham group. These differences were not statistically significant. Only one eye in the DEX implant-treated group required cataract surgery at the end of the 26-week study. Other adverse effects included: conjunctival hemorrhage, ocular discomfort, eye pain, and iridocyclitis. The between groups analysis for adverse effects showed no statistically significant difference between treated (DEX implant) and nontreated eyes (sham). There were no systemic side effects noted in the DEX implant groups. The authors concluded that a single dose of the DEX implant was well tolerated in patients with noninfectious posterior uveitis with significant improvement in intraocular inflammation and visual acuity at 26-week follow-up. The $0.7 \mathrm{mg}$ DEX implant demonstrated greater efficacy than the $0.35 \mathrm{mg}$ implant with a similar safety profile.

\section{Advantages and disadvantages of the dexamethasone intravitreal implant}

The use of the DEX intravitreal implant has shown significant promise for the treatment of macular edema and more recently noninfectious intermediate and posterior uveitis.

It is difficult to compare the efficacy and safety profile of the DEX intravitreal implant with other sustained-release corticosteroid implants, specifically the FA implant, because there are currently no randomized controlled trials to compare these devices (Table 2). However, in terms of outcomes of BCVA, the improvement of 15 letters or more in the FA implant was $21 \%$ at 34 weeks. In comparison, $38 \%$ of eyes attained 15 -letter improvement in the DEX implant study at 26 weeks. These findings could indicate an improved response in terms of visual outcomes with the
DEX implant in comparison to the FA implant although it is difficult to draw conclusions given the different half-lives of the two implants. It is also difficult to compare adverse effect outcomes without a head-to-head randomized control trial given the different pharmacokinetic properties and duration of effect of the two corticosteroid implants. There is no comparative data analyzing secondary adverse effect outcomes at 6 months in patients who have received an FA implant. Given the available data, the FA implant has significant ocular adverse outcomes requiring additional eye surgery for the management of cataracts and glaucoma after 30 months. These findings are in comparison to the DEX implant data where few patients required additional ocular surgery for the management of adverse effects after 6 months. As previously discussed it may also be a result of the lipophilicity of the corticosteroid used; FA has greater portioning into the lens and trabecular meshwork in comparison to dexamethasone and this may account for its higher side-effect profile. ${ }^{21}$ Longer duration of exposure of ocular structures to steroids may also play a role in adverse effects and the safety profile of different steroid moieties may also be time dependent. However, chronic ocular inflammation may require repeated DEX implantation. Long-term data is necessary and further longitudinal studies on the DEX implant need to be performed in order to be able to effectively compare adverse effects to other longer-acting steroid implants.

There are many advantages to the DEX implant system in comparison to other steroid implants. First, the biodegradable matrix used in the DEX implant in comparison to the surgically implanted polymer in the FA implant is of major importance. The biodegradable polymer provides significant clinical benefit in terms of ease of sequential implants without the need to surgically remove older devices. Second, the platform for delivery has been devised so that it can be performed in an outpatient setting as a more routine intravitreal injection as opposed to a surgical procedure which significantly

Table 2 Comparison of intravitreal implants for the treatment of noninfectious uveitis

\begin{tabular}{|c|c|c|}
\hline & $\begin{array}{l}0.59 \mathrm{mg} \text { fluocinolone acetonide } \\
\text { (FA) implant }\end{array}$ & $\begin{array}{l}0.7 \mathrm{mg} \text { dexamethasone } \\
\text { (DEX) implant }\end{array}$ \\
\hline Administration & Operating room & Office-based \\
\hline Matrix & Non-biodegradable & Biodegradable \\
\hline Duration of effect & 30 months & 6 months \\
\hline Improvement of $>15$ letters (\% eyes) & $21 \%$ by week 34 & $38 \%$ by week 26 \\
\hline Rescue medications (\% eyes) & $25.4 \%$ by week 34 & $22 \%$ by week 26 \\
\hline Glaucoma surgery (\% eyes) & $30.6 \%$ by month 24 & $0.5 \%$ by month 6 \\
\hline \multirow[t]{2}{*}{ Cataract surgery (\% eyes) } & $89.4 \%$ by month 24 & $4 \%$ by month 6 \\
\hline & $100 \%$ by month 30 & \\
\hline
\end{tabular}


lowers the cost of implantation. Finally, the cost of the DEX implant system is significantly lower than the cost of the FA implant by approximately $65 \%$. This cost approximation also does not take into consideration the additional costs of secondary surgeries such as cataract surgery and glaucoma filtering procedures that may be necessary in a large portion of the FA implant patient population. However, the DEX implant system may require multiple implantations per year which can escalate its cost. Again, further longitudinal studies on the DEX implant need to be performed to be able to effectively compare the cost-benefit ratio when compared to other longeracting steroid implants.

\section{Future applications}

More recently, animal studies have been used to evaluate the use of a sustained dexamethasone implant for the treatment of experimental anterior and intermediate uveitis. ${ }^{31}$ Ghosn et al assessed the efficacy of the implant in a rabbit model of anterior and intermediate uveitis. Inflammation was induced using unilateral injection of Mycobacterium tuberculosis $\mathrm{H} 37 \mathrm{Ra}$ antigen in preimmunized rabbits. ${ }^{31}$ Four days after induction of inflammation, the rabbits received an intravitreal dexamethasone device or underwent sham procedure. Clinical and histopathological signs were assessed at 13 days. In addition, inflammatory markers were measured in the iris and ciliary body at 21 days. The authors found that both clinical and histopathological signs of anterior and intermediate uveitis were significantly decreased in eyes that had received the implant compared with sham eyes. Inflammatory cytokines were also significantly decreased in the iris and ciliary body of eyes that had been treated, indicating a reduced intraocular inflammatory response. The authors concluded that posterior drug delivery of a sustained release dexamethasone implant also achieves therapeutic levels of drug in the anterior chamber and pars plana. Further clinical studies are needed to evaluate safety and efficacy in treating these entities in humans.

Other future considerations in understanding the role of inflammation in eye disease are the use of corticosteroid implants in patients with age-related macular degeneration (AMD). A study involving use of the DEX implant in patients with AMD was performed to evaluate its benefits as adjunctive therapy to ranibizumab for the treatment of choroidal neovascularization in AMD. ${ }^{32}$ In a randomized, controlled trial of 243 eyes, there was a significant increase in the time between doses of "as needed" injections of ranibizumab with longer ranibizumab injection-free intervals in patients who received the DEX implant. In addition, the authors found that addition of the DEX implant increased the percentage of eyes with retinal thickness $<250 \mu \mathrm{m}$. Adverse effects were similar to those seen in other DEX implant trials.

\section{Conclusions and place in therapy}

There is significant morbidity associated with uveitis, specifically posterior uveitis which can account for $10 \%$ of cases of legal blindness in the United States. Corticosteroids remain the mainstay of treatment of all forms of uveitis but have significant adverse effects. The dexamethasone drug delivery system is a novel approach to the treatment of uveitis and has been shown to be efficacious in the treatment of noninfectious uveitis with potentially fewer adverse effects than other steroid moieties. As a result of recent clinical trials, the $0.7 \mathrm{mg}$ dexamethasone sustained release intravitreal implant has been FDA approved for both the treatment of macular edema due to retinal vein occlusions and also for the treatment of noninfectious, posterior uveitis. Future studies will need to determine the relative efficacy and safety profiles among the different intravitreal steroid implants and the long-term effects of repeated use of the dexamethasone implant in the treatment of recurrent uveitis. Overall, the dexamethasone implant is an important new treatment modality and initial studies have demonstrated its efficacy with minimal adverse effects in the treatment of noninfectious uveitis.

\section{Disclosure}

The authors report no conflicts of interest in this work.

\section{References}

1. Albert DM, Jakobiec FA, editors. Principles and Practice of Ophthalmology. 2nd ed. Philadelphia, PA: WB Saunders Co; 2000.

2. Bloch-Michel E, Nussenblatt RB. International Uveitis Study Group recommendations for the evaluation of intraocular inflammatory disease. Am J Ophthalmol. 1987;103(2):234-235.

3. Jabs DA, Akpek EK. Immunosuppression for posterior uveitis. Retina. 2005;25(1):1-18.

4. Taylor SR, Isa H, Joshi L, Lightman S. New developments in corticosteroid therapy for uveitis. Ophthalmologica. 2010;224 Suppl 1:46-53.

5. Leopold IH, Purnell JE, Cannon EJ, Steinmetz CG, McDonald PR. Local and systemic cortisone in ocular disease. Am J Ophthalmol. 1951;34(3): 361-371.

6. Yeh S, Nussenblatt RB. Fluocinolone acetonide for the treatment of uveitis: weighing the balance between local and systemicimmunosuppression. Arch Ophthalmol. 2008;126(9):1287-1289.

7. Kiernan DF, Mieler WF. The use of intraocular corticosteroids. Expert Opin Pharmacother. 2009;10(15):2511-2525.

8. Kok H, Lau C, Maycock N, McCluskey P, Lightman S. Outcome of intravitreal triamcinolone in uveitis. Ophthalmology. 2005;112(11): 1916. e1-e7.

9. van Kooij B, Rothova A, de Vries P. The pros and cons of intravitreal triamcinolone injections for uveitis and inflammatory cystoid macular edema. Ocul Immunol Inflamm. 2006;14(2):73-85. 
10. Nguyen QD, Callanan D, Dugel P, Godfrey DG, Goldstein DA, Wilensky JT. Treating chronic noninfectious posterior segment uveitis: the impact of cumulative damage. Proceedings of an expert panel roundtable discussion. Retina. 2006;Suppl:1-16.

11. Lobo AM, Sobrin L, Papaliodis GN. Drug delivery options for the treatment of ocular inflammation. Semin Ophthalmol. 2010;25(5-6): 283-288.

12. Jaffe GJ, Pearson PA, Ashton P. Dexamethasone sustained drug delivery implant for the treatment of severe uveitis. Retina. 2000;20(4):402-403.

13. Jaffe GJ, Ben-Nun J, Guo H, Dunn JP, Ashton P. Fluocinolone acetonide sustained drug delivery device to treat severe uveitis. Ophthalmology. 2000;107(11):2024-2033.

14. Brumm MV, Nguyen QD. Fluocinolone acetonide intravitreal sustained release device - a new addition to the armamentarium of uveitic management. Int J Nanomedicine. 2007;2(1):55-64.

15. Jaffe GJ, Martin D, Callanan D, Pearson PA, Levy B, Comstock T. Fluocinolone acetonide implant (Retisert) for noninfectious posterior uveitis: thirty-four-week results of a multicenter randomized clinical study. Ophthalmology. 2006;113(6):1020-1027.

16. Callanan DG, Jaffe GJ, Martin DF, Pearson PA, Comstock TL. Treatment of posterior uveitis with a fluocinolone acetonide implant: three-year clinical trial results. Arch Ophthalmol. 2008;126(9):1191-1201.

17. Jaffe GJ. Reimplantation of a fluocinolone acetonide sustained drug delivery implant for chronic uveitis. Am J Ophthalmol. 2008;145(4): 667-675.

18. Goldstein DA, Godfrey DG, Hall A, et al. Intraocular pressure in patients with uveitis treated with fluocinolone acetonide implants. Arch Ophthalmol. 2007;125(11):1478-1485.

19. Sivaprasad S, McCluskey P, Lightman S. Intravitreal steroids in the management of macular oedema. Acta Ophthalmol Scand. 2006;84(6): $722-733$.

20. Edelman JL. Differentiating intraocular glucocorticoids. Ophthalmologica. 2010;224 Suppl 1:25-30.

21. Thakur A, Kadam R, Kompella UB. Trabecular meshwork and lens partitioning of corticosteroids: implications for elevated intraocular pressure and cataracts. Arch Ophthalmol. 2011;129(7):914-920.
22. Sawyer AJ, Saucier-Sawyer JK, Booth CJ, et al. Convection-enhanced delivery of camptothecin-loaded polymer nanoparticles for treatment of intracranial tumors. Drug Deliv Transl Res. 2011;1(1):34-42.

23. London NJ, Chiang A, Haller JA. The dexamethasone drug delivery system: indications and evidence. Adv Ther. 2011;28(5):351-366.

24. Chang-Lin JE, Attar M, Acheampong AA, et al. Pharmacokinetics and pharmacodynamics of a sustained-release dexamethasone intravitreal implant. Invest Ophthalmol Vis Sci. 2011;52(1):80-86.

25. Chang-Lin JE, Burke JA, Peng Q, et al. Pharmacokinetics of a sustainedrelease dexamethasone intravitreal implant in vitrectomized and nonvitrectomized eyes. Invest Ophthalmol Vis Sci. 2011;52(7):4605-4609.

26. Cheng CK, Berger AS, Pearson PA, Ashton P, Jaffe GJ. Intravitreal sustained-release dexamethasone device in the treatment of experimental uveitis. Invest Ophthalmol Vis Sci. 1995;36(2):442-453.

27. Kuppermann BD, Blumenkranz MS, Haller JA, et al. Randomized controlled study of an intravitreous dexamethasone drug delivery system in patients with persistent macular edema. Arch Ophthalmol. 2007; 125(3):309-317.

28. Williams GA, Haller JA, Kuppermann BD, et al. Dexamethasone posterior-segment drug delivery system in the treatment of macular edema resulting from uveitis or Irvine-Gass syndrome. Am J Ophthalmol. 2009;147(6):1048-1054.

29. Haller JA, Bandello F, Belfort R Jr, et al. Randomized, sham-controlled trial of dexamethasone intravitreal implant in patients with macular edema due to retinal vein occlusion. Ophthalmology. 2010;117(6): 1134-1146.

30. Lowder C, Belfort R Jr, Lightman S, et al. Dexamethasone intravitreal implant for noninfectious intermediate or posterior uveitis. Arch Ophthalmol. 2011;129(5):545-553.

31. Ghosn CR, Li Y, Orilla WC, et al. Treatment of experimental anterior and intermediate uveitis by a dexamethasone intravitreal implant. Invest Ophthalmol Vis Sci. 2011;52(6):2917-2923.

32. Kupperman B. Safety and efficacy of dexamethasone intravitreal implant in adjunctive therapy to Lucentis in patients with choroidal neovascularization secondary to age-related macular degeneration. 33rd Annual Macular Society Meeting; February 24, 1010; Tucson, AZ.
Clinical Ophthalmology

\section{Publish your work in this journal}

Clinical Ophthalmology is an international, peer-reviewed journal covering all subspecialties within ophthalmology. Key topics include: Optometry; Visual science; Pharmacology and drug therapy in eye diseases; Basic Sciences; Primary and Secondary eye care; Patient Safety and Quality of Care Improvements. This journal is indexed on

\section{Dovepress}

PubMed Central and CAS, and is the official journal of The Society of Clinical Ophthalmology (SCO). The manuscript management system is completely online and includes a very quick and fair peer-review system, which is all easy to use. Visit http://www.dovepress.com/ testimonials.php to read real quotes from published authors. 